\title{
The Milch Technique for reduction of anterior shoulder dislocations in an accident and emergency department.
}

\section{G. JOHNSON, W. HULSE \& A. MCGOWAN}

Accident and Emergency Department, Pinderfields General Hospital, Wakefield, West Yorks

\section{SUMMARY}

Following the introduction of the Milch technique as the preferred method of reduction of anterior shoulder dislocations in an $A \& E$ department, a retrospective study of the technique was carried out.

A total of 187 patients with anterior shoulder dislocations were included. The Milch technique was attempted in 142 cases with a success rate of $86 \%$. There was no statistical difference in the success rates of junior and senior staff. In $73 \%$ of the Milch cases either no analgesia or sedation or Entonox alone was used.

This study shows that the Milch technique can successfully be used by inexperienced staff to reduce dislocated shoulders with a reduction in the requirements for sedation and anaesthetics.

\section{INTRODUCTION}

In recent years a number of reports have been published advocating the Milch technique as a method of reducing anterior glenohumeral dislocation in preference to the established Kocher and Hippocratic methods (Russell et al., 1981; Beattie et al., 1986). The Milch technique is reported to be relatively atraumatic and painless with a success rate equal to the more established methods. In these studies it was carried out by a restricted number of experienced staff.

We studied the use of the Milch technique in the Accident and Emergency Department at Pinderfields General Hospital to determine its suitability in this setting particularly with respect to the success rates of junior medical staff and the use of analgesia, sedation and anaesthesia.

Correspondence: $\mathrm{Mr}$ G. Johnson FRCS, Registrar in Accident and Entergency, Hull Royal Infirmiary, Anlaby Rd, Hull, Humberside, HU3 2JZ. 


\section{METHODS}

A series of 187 consecutive patients with anterior shoulder dislocations presenting to the Accident and Emergency Department at Pinderfields Hospital between August 1985 and March 1990 were studied. Patients with fractures of the greater tuberosity were included but other types of fracture-dislocation were excluded.

Junior medical staff were instructed how to perform the Milch technique by either demonstration on a patient or by watching a video of the method. The Milch manoeuvre is performed by initially abducting the arm then applying external rotation. Reduction is achieved by a combination of gentle axial traction and direct pressure over the humeral head via the axilla (Milch, 1938).

Records were examined retrospectively to determine the method of reduction used and its success. Data were also collected on the use of analgesia, sedation or anaesthetic and the grade of doctor performing the manipulation.

\section{RESULTS}

A total of 187 patients with anterior shoulder dislocation were identified during the study period. In 142 cases the Milch technique was used initially. Of the remaining cases, 13 were referred for in-patient management due to associated injuries and in the final 32 the doctor responsible elected to use an alternative method of reduction, either due to unfamiliarity with the Milch technique or the inability of the patient to cooperate due to intoxication.

The Milch technique was successful in 122 out of 142 attempts, a success rate of $86 \%$. The grade of the doctor performing the manipulation is shown in Fig. 1. When the success rate was analysed by grade of staff (Fig. 2) no statistically significant difference was found although there is a tendency for a higher success rate amongst the most senior staff. One consultant achieved a personal success rate of $93 \%$. Of

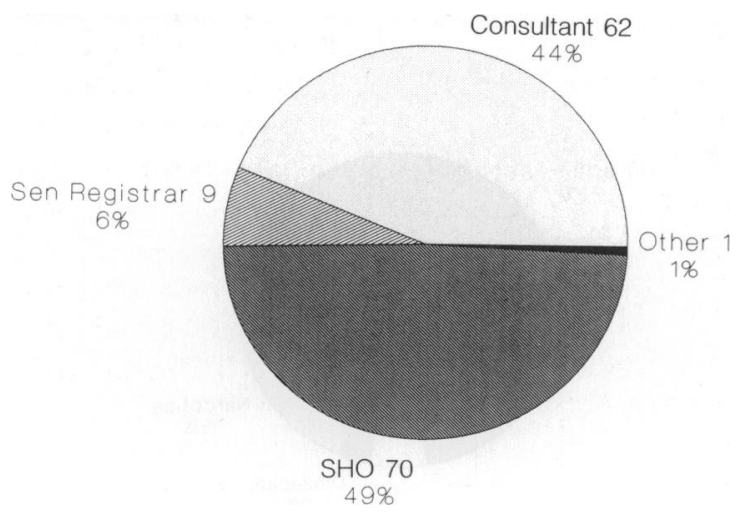

Fig. 1. Attempted Milch reduction by grade of staff. Total 142 patients. 


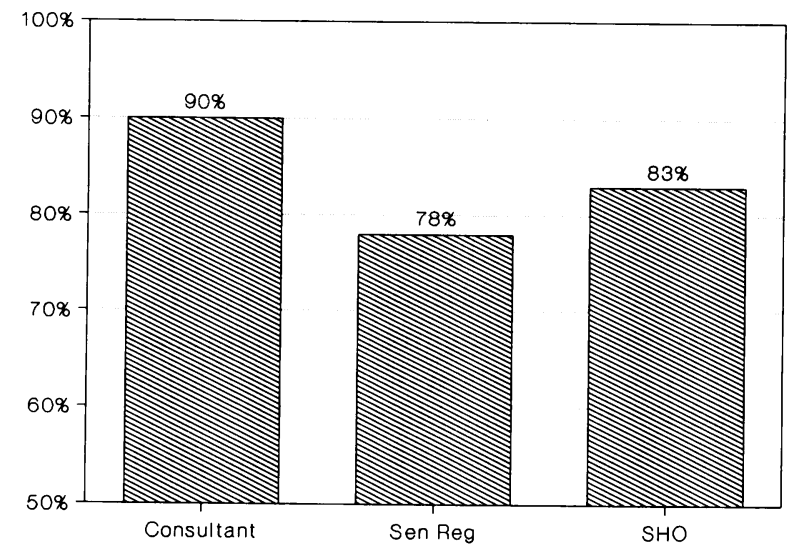

Fig. 2. Success rate for Milch reductions by grade of staff.

particular note are the 70 reductions which were performed by a total of 35 different A\&E senior house officers (SHOs) with a success rate of $83 \%$. No complications due to the manipulations were identified.

When the Milch technique was used, $73 \%$ of patients required only Entonox or no analgesia or sedation (Fig. 3). All six patients with an associated fracture of the greater tuberosity required either analgesia or anaesthetic.

\section{DISCUSSION}

Anterior glenohumeral dislocation is a common injury presenting to A\&E departments. It often occurs in sporting competition or in association with alcohol intoxication and patients therefore frequently present at nights or weekends when less senior medical staff are available. At present many departments reduce shoulder

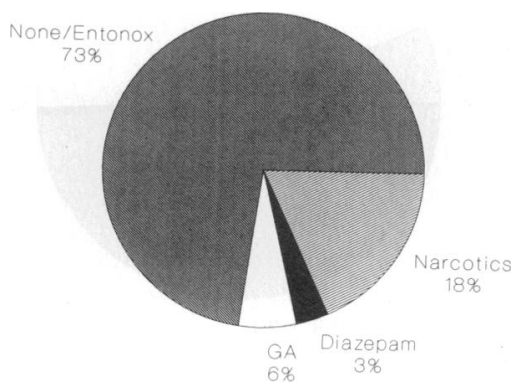

Fig. 3. Drugs used for reductions by Milch technique. 
dislocations either under a general anaesthetic or with the aid of parenteral analgesia or sedation. This requires the use of further staff during the procedure and afterwards to observe recovery. Manipulation without sedatives or anaesthetic allows rapid patient recovery thus reducing the time the patient spends in the department and freeing medical and nursing staff for other tasks.

We have shown that in everyday use in an A\&E department, the Milch technique is safe, has a high success rate and can be applied in most cases without the need for analgesia or anaesthesia. This success rate can be maintained even when reductions are carried out by junior staff with little previous experience of the technique provided they are suitably instructed.

\section{REFERENCES}

Beattie T. F., Steedman D. J., Mc Gowan A. \& Robertson C. E. (1986) A comparison of the Milch and Kocher techniques for acute anterior dislocation of the shoulder. Injury 17, 349-352.

Milch H. (1938) Dislocation of the shoulder. Surgery 3, 732-738.

Russell J. A., Holmes E. M. \& Keller D. J. (1981) Reduction of anterior shoulder dislocation using the Milch technique: study of ski injuries. Journal of Trauma 21, 802-806. 